

\section{DISCLAIMER}

This report was prepared as an account of work sponsored by an agency of the United States Government. Neither the United States Government nor any agency Thereof, nor any of their employees, makes any warranty, express or implied, or assumes any legal liability or responsibility for the accuracy, completeness, or usefulness of any information, apparatus, product, or process disclosed, or represents that its use would not infringe privately owned rights. Reference herein to any specific commercial product, process, or service by trade name, trademark, manufacturer, or otherwise does not necessarily constitute or imply its endorsement, recommendation, or favoring by the United States Government or any agency thereof. The views and opinions of authors expressed herein do not necessarily state or reflect those of the United States Government or any agency thereof. 


\section{DISCLAIMER}

Portions of this document may be illegible in electronic image products. Images are produced from the best available original document. 
UCRL- 17795

UC- 34 Physics

TID- 4500 (50th Ed.)

\section{UNIVERSITY OF CALIFORNIA}

Lawrence Radiation Laboratory

Berkeley, California

AEC Contract No. W-7405-eng-48

SOME THOUGHTS ON STABILITY

IN NONLINEAR PERIODIC FOCUSING SYSTEMS

Edwin M. McMillan

September 5, 1967

\section{LEGAL NOTICE}

This report was prepared as an account of Government sponsored work Neither the United States, nor the Commission, nor any person acting on behalf of the Commission

A Makes any warranty or representation, expressed or implied, with respect to the accuracy, completeness, or usefulness of the information contuined in this report, or that the use of any information, apparatus, method, or process disclosed in this report may not infringe of any lnformation, apparatu

B. Assumes any labilities with respect to the use of, or for damages resulting from the use of any information, apparatus, method, or process disclosed in this report.

As used in the above, "person acting on behalf of the Commission" includes any employee or contractor of the Commission, or employee of such contractor, to the extent that such employee or contractor of the Commlesion, or employee of such contractor prepares, disseminates, or provides access to, any information pursuant to his employment or contract with the Commission, or his empioyment with such contractor. 



\title{
SOME THOUGHTS ON STABILITY \\ IN NONLINEAR PERIODIC FOCUSING SYSTEMS
}

\author{
Edwin M. McMillan \\ Lawrence Radiation Laboratory \\ University of California \\ Berkeley, California
}

September 5, 1967

\begin{abstract}
A brief discussion is given of the long-term stability of particle motions through periodic focusing structures containing lumped nonlinear elements. A method is presented whereby one can specify the nonlinear elements in such a way as to generate a variety of structures in which the motion has long-term stability.
\end{abstract}




\section{Introduction}

The stability of the motion of a particle through a periodically repeated focusing system (or of a light ray through a repeated sequence of lenses) poses a difficult and fascinating problem. Let us imagine a set of planes intersecting the path and spaced with the same periodicity as the system, and examine the coordinates of the deflection at these planes. One degree of freedom will be considered, with the coordinate $x$ and the conjugate momentum $y$. (The neglect of coupling with other degrees of freedom is already an oversimplification of the real physical problem.) As we go from one plane to the next, the new values $x^{i}, y^{\prime}$ are given as functions of $x, y$. If the equations of motion are derivable from a Hamiltonian, the Jacobian of this transformation is unity; this is assumed. (Effects of scattering and radiation damping are thereby excluded.) We now look for fixed points of the transformation, that is, for values of $\mathrm{x}, \mathrm{y}$ for which $\mathrm{x}^{\prime}=\mathrm{x}, \mathrm{y}^{\prime}=\mathrm{y}$. In the immediate vicinity of a fixed point, the transformation can be linearized to

$$
\begin{aligned}
& x^{\prime}=a x+b y, \quad(a d-b c=1) \\
& y^{\prime}=c x+d y,
\end{aligned}
$$

The results of the continued iteration of (1) are well known. Stability is determined by $a+d$, the trace of the transformation matrix. Three cases can occur:

(a) If $-2<(a+d)<2$, the motion is stable, and points in the phase plane tend to move around the fixed point. If $n$ is the number of iterations, $x$ and $y$ can be expressed as linear combinations of $\cos \mu n$ and $\sin \mu n$, with $2 \cos \mu=a+d$.

(b) If $(a+d)>2$, the motion is unstable, and points tend to move away from or toward the fixed point. In this case, $x$ and $y$ can be expressed as linear combinations of $e^{\lambda n}$ and $e^{-\lambda n}$, with $2 \cosh \lambda=a+d$.

(c) If $(a+d)<-2$, the motion is again unstable, but with a superimposed oscillation; $x$ and $y$ can be expressed as linear combinations of $(-1)^{\mathrm{n}} \mathrm{e}^{\lambda \hat{\mathrm{n}}}$ and $(-1)^{\mathrm{n}} \mathrm{e}^{-\lambda \mathrm{n}}$, with $-2 \cosh \lambda=\mathrm{a}+\mathrm{d}$. This will be called an oscillatory instability.

In cases (b) and (c) two lines can be drawn through the fixed point, which have the property that points on these lines remain on them, that is, $y^{\prime} / x^{\prime}=y / x$. The slopes of these lines (i.e., $y / x$ ) are given by the two values of $\frac{1}{2 b}\left(d-a \pm \sqrt{\left.(a+d)^{2}-4\right)}\right.$; the product of the two values is $-c / b$. Looking at the lines as broken into segments by the fixed points, we have four directions to consider: along one pair of opposite directions, phase points are moving away from the fixed point, and along the other pair they are approaching it asymptotically. These lines are called invariant lines. 
When we move into the cold nonlinear world beyond the linearized region, the invariant lines persist. Points along a short linear segment transform into points beyond, allowing extension of the line; these go to still farther points, and so on. (Invariant lines on which the points move inward can be extended by using the inverse transformation.) Because the transformation is single-valued, an invariant line can never cross itself. It can proceed to infinity, or it can join smoothly onto another invariant line having the same sense of motion of phase points; this other line can originate from the same fixed point from which the first line started, or from a different one. Or it can cross a different invariant line; then something very complicated happens. The crossing point is common to both lines; the transformation must carry this point to both lines, so they must cross again, and this must be repeatable indefinitely on both directions. Furthermore, the lines at corresponding intersections must cross in the same sense; therefore there must be another crossing point between, which must also be repeated. A dollar sign with a single bar gives a picture of the behavior for three successive crossings. The unity Jacobian requires preservation of areas, therefore all the loops on the same side must have the same area. A symmetry which will be discussed later assures that at one crossing point the adjacent loops are mirror images, therefore all loops have the same area. As the infinite sequence of crossing points moves toward a fixed point, the crossing points get very close together, and the loops get very long and thin.

What is the relation of all this to stability? It can be shown that, if a stable fixed point is completely surrounded by smoothly joined invariant lines, all points inside the enclosure will remain inside indefinitely, guaranteeing stability under an unlimited number of iterations of the transformation. On the other hand, if the invariant lines cross, the re will be a breach in the wall. Loops which enter the interior, where they curl up in a complicated fashion as they get longer and thinner, will on later iterations find a way through the breach and carry points outside. This process may, however, require a very large number of iterations, and situations which are in principle unstable may be stable for practical purposes.

Various questions can be asked: What determines the smooth joining of invariant lines? Can one find nonlinear focusing systems where the smooth joining is guaranteed? In case a smooth join is not achieved, do the loops always invade the whole interior, or can a finite sanctuary remain? If it is achieved, will all or some of the points in the interior be confined to motion on smooth closed curves?

In the work that I have seen on this subject, the nonlinear transformation has been simplified to a form which represents physically a lumped nonlinear impulse applied at a single plane in each repeat unit of an otherwise linear focusing system. An example is the transformation introduced by Professor de Vogelaere:

$$
\begin{aligned}
& x^{\prime}=y+x^{2} \\
& y^{\prime}=-x+x^{\prime 2}=-x+\left(y+x^{2}\right)^{2}
\end{aligned}
$$


Extensive numerical calculations with this transformation have given valuable insight into the behavior of the invariant lines when the invariant lines cross at a large angle, making loops of considerable area. L. Jackson Laslett has investigated another form with a quadratic nonlinear term, which gives very small loops. I wish to thank both these men for introducing me to this subject, and for many discussions and arguments about it.

\section{The Transformation Used}

I started with the form

$$
\begin{aligned}
& x^{\prime}=y, \\
& y^{\prime}=-x+f(y)
\end{aligned}
$$

mainly on the grounds that it is simple, and that it allows the introduction of an arbitrary function $f(y)$ without disturbing the unity value of the Jacobian. (Later I realized that de Vogelaere's and Laslett's forms could be converted to this form by a coordinate transformation.) For physical justification, one can start with a linear system represented by (1). Let the spacing between measurement planes be taken as unity. Into this system the nonlinear impulse is added by introducing a thin nonlinear lens immediately before each measurement plane. This lens is specified by the change in slope of path $y$ when a particle passesthrough at a displacement $\mathrm{x}$ :

$$
\Delta y=-(a+d) x+f(x)
$$

In each section, the particle passes through the lens just before it leaves the section, so that the first equation of (1) remains unchanged while $\Delta y$ from (4) (as a function of $x^{\prime}$ ) is added to the right side of the second equation. Now make the coordinate transformation $\mathrm{X}=\mathrm{x}, \mathrm{Y}=\mathrm{ax}+\mathrm{by}$. This leads to

$$
\begin{aligned}
& X^{\prime}=Y \\
& Y^{\prime}=-X+(a+d) Y-(a+d) X^{\prime}+f\left(X^{\prime}\right)
\end{aligned}
$$

Substituting $X^{\prime}$ from the first into the second equation, and reverting to the lower-case notation for the variables, we get Eq. (3). The inverse transformation can be obtained by replacing $x^{\prime}$ with $y, y^{\prime}$ with $x, x$ with $\mathrm{y}^{\prime}$, and $\mathrm{y}$ with $\mathrm{x}^{\prime}$ in (3).

[For further physical insight, note that (3) can represent a linear thin lens with $\Delta y=-x$ placed just after the measurement plane, plus a lens with $\Delta y=-x+f(x)$ placed just before it. The combined effect of these lenses is $\Delta y=-2 x+f(x)$. Thus $f(x)=2 x$ corresponds to free motion of the particle. The resulting transformation looks unfamiliar because $y$ is measured between two canceling lenses in immediate contact. 
If we introduce $Y=$ slope outside the gap between lenses, then $y=Y+x$, and the transformation takes the familiar form $x^{\prime}=x+Y, \quad Y^{\prime}=Y$.]

\section{A Useful Property of the Transformation}

The great virtue of the transformation in the form (3) is the property to be described, which allows one to construct lens functions $f(x)$ leading to prescribed invariant lines $x=\phi(y)$. If a point lying on the line $\mathbf{x}=\phi(y)$ is subjected to the transformation, we get

$$
\begin{aligned}
& x^{\prime}=y, \\
& y^{\prime}=-\phi(y)+f(y) .
\end{aligned}
$$

Now we require that $x^{\prime}=\phi\left(y^{\prime}\right)$, the condition that the transformed point lies on the same line. Write this in the inverted form $y^{\prime}=\phi^{-1}\left(x^{\prime}\right)$, and substitute $x^{\prime}$ and $y^{\prime}$ from (6), giving

$$
f(y)=\phi(y)+\phi^{-1}(y) \text {. }
$$

Therefore if we use for the lens function $f$ the sum of any function and its inverse, both the function and its inverse are invariant lines of the resulting transformation.

This leads to a simple geometrical construction. The inverse of $\mathrm{x}=\phi(\mathrm{y})$ is obtained by interchanging $\mathrm{x}$ and $\mathrm{y}$ and solving for $\mathrm{x}$. The interchange corresponds to a reflection about the positive diagonal $\mathrm{x}=\mathrm{y}$. An arbitrary monotonic curve crossing the diagonal in two points can now be drawn and its reflection constructed. The midpoints of horizontal segments joining the curve and its reflection lie on $x=\frac{1}{2} f(y)$. Because of the symmetry, $y=\frac{1}{2} f(x)$ is given by the line of midpoints of vertical segments. Fixed points are at $f(x)=2 x$, that is, at the intersections of $\frac{1}{2} f(x)$ with the diagonal. The trace of the matrix of the linearized transformation at an intersection is equal to $d f(x) / d x$. If $\frac{1}{2} f(x)$ crosses the diagonal in the upward direction, the fixed point is unstable, if downward, stable, unless the slope lies below that of the negative diagonal, when oscillatory instability occurs. An example based on the rectangular hyperbolas $\phi(x)=1-a /(x+1)$,
$\phi^{-1}(x)=-1-a /(x-1)$, with $f(x)=2 a x /\left(1-x^{2}\right)$, is plotted in Fig. 1, for $a=\frac{1}{2}$. The arrows show the results of the transformation applied to various points. A point starting at the intersection of $y=f(x)$ with $x=\frac{1}{2} f(y)$ returns to the same place after 8 iterations. Similarly, a point starting at the intersection of $y=f(x)$ with the diagonal would return after 6 iterations, but this point has moved to the origin with the choice of $a=\frac{1}{2}$. The value of $\mu$ at the stable fixed point is $\pi / 3$. 


\section{A Proof of Unlimited Stability}

The form of (3) is such that a point lying on a given horizontal line transforms to a point lying on a vertical line intersecting the horizontal line at the diagonal. Displacement of the first point to the right leads to an equal downward displacement of the second. In Fig. 1, A and B are two such points on an invariant line. By the symmetry of the figure, the segments $A A^{\prime}$ and $B^{\prime} B^{\prime}$ are of equal length. Therefore any point on $\mathrm{AA}^{\prime}$ transforms to a point on $\mathrm{BB}^{\prime}$, and all points lying in the region enclosed by the two invariant lines remain inside this region

\section{Closed Curves}

In Section 3 it was specified that $\phi$ should be a monotonic function. If this is not true, its inverse is multivalued. The condition $f=\phi+\phi^{-1}$ must be satisfied on both branches, which requires the addition of another branch to $\phi$. This leads to a second intersection between $\phi$ and $\phi^{-1}$, and we are drawn inexorably into the complication of an infinite series of intersections. This difficulty does not occur if $\phi$ and $\phi^{-1}$ join smoothly at their intersection on the diagonal. The portion of $\phi$ on one side of the diagonal can then be combined with the portion of $\phi^{-1}$ on the other into a single curve which is its own inverse, and the function $\frac{1}{2} f(x)$ is given by the line of midpoints of segments between the upper and lower branches. (Curves that are reentrant in such a way that they are more than doublevalued require satisfaction of $\mathrm{f}=\phi+\phi^{-1}$ on all branches. Such curves can exist without leading to intersections, but they cannot be drawn arbitrarily.) Cases like those illustrated in Fig. 2 can occur. In case $2 \mathrm{~b}$, the closed curve does not pass through a fixed point and is not an invariant line in the "classical" sense. There is no place where the transformation leads to an immediately adjacent point, and therefore no way to establish an initial direction for computing the further progress of the line. Nevertheless, it is invariant in the sense that points remain on it indefinitely, and it is equally effective in ensuring that interior points remain inside, giving indefinite stability.

As an example, we can use the central conics. Let $\mathrm{X}, \mathrm{Y}$ be coordinates parallel and perpendicular to the positive diagonal. The conic $\mathrm{Y}^{2}=\mathrm{a}+\mathrm{bX}^{2}$, expressed in terms of $\mathrm{x}$ and $\mathrm{y}$, is

$$
y=\frac{1}{1-b}\left[(1+b) x \pm \sqrt{4 b x^{2}+2 a(1-b)}\right] \text {. }
$$

The function $y=f(x)$ is given by the sum of the two branches,

$$
y=2 \frac{1+b}{1-b} x
$$




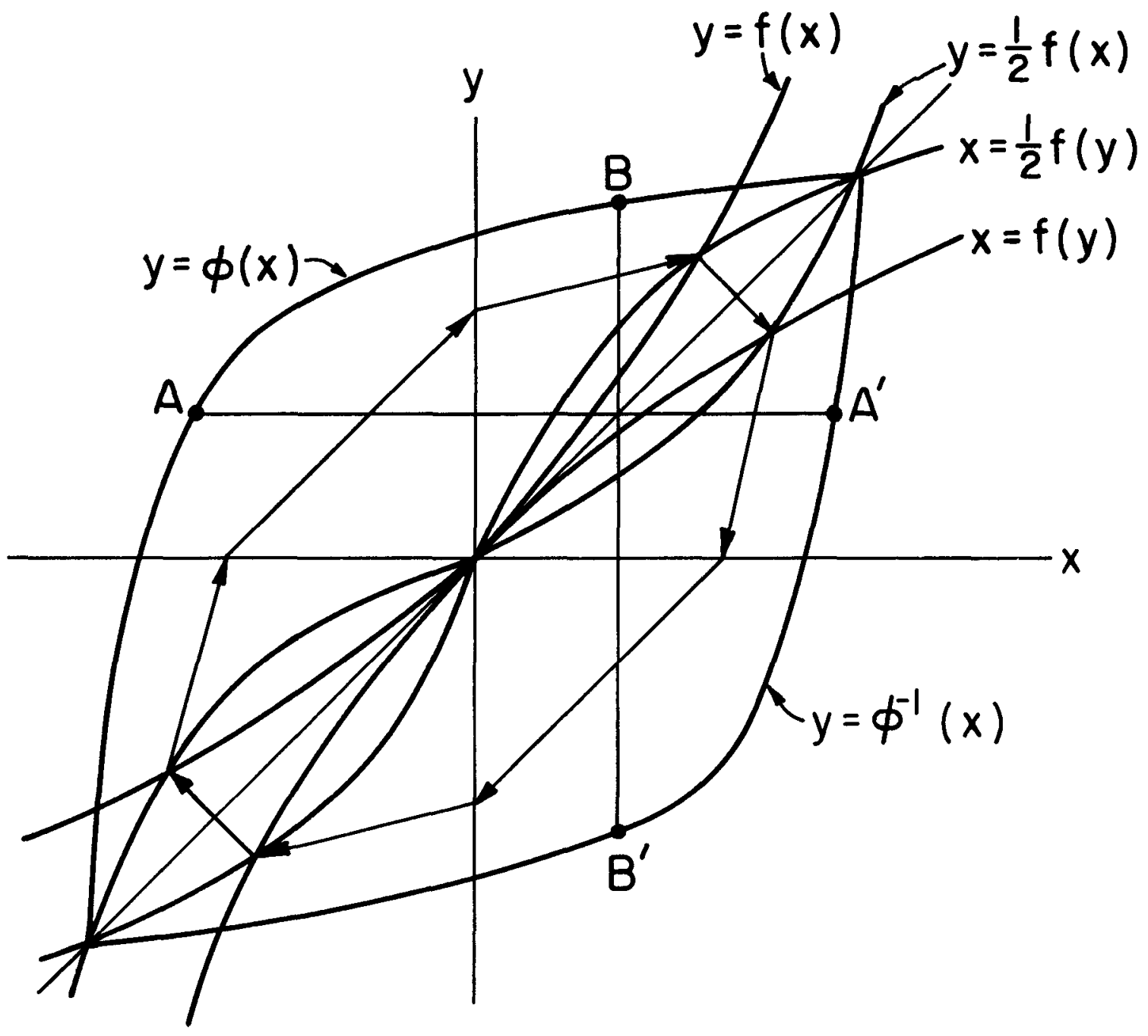

XBL679-5216

Fig. 1 


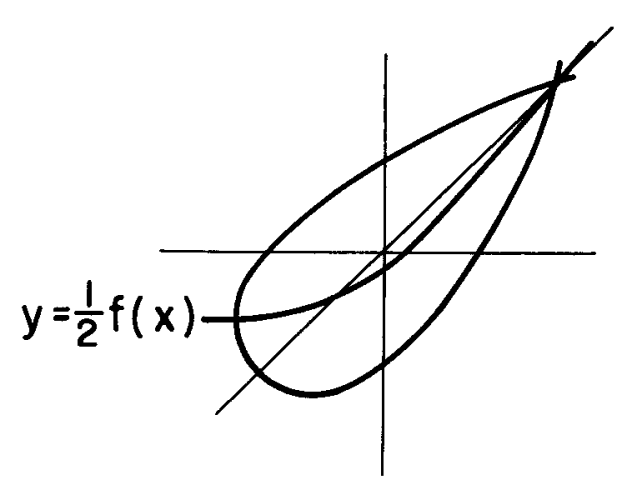

(a)

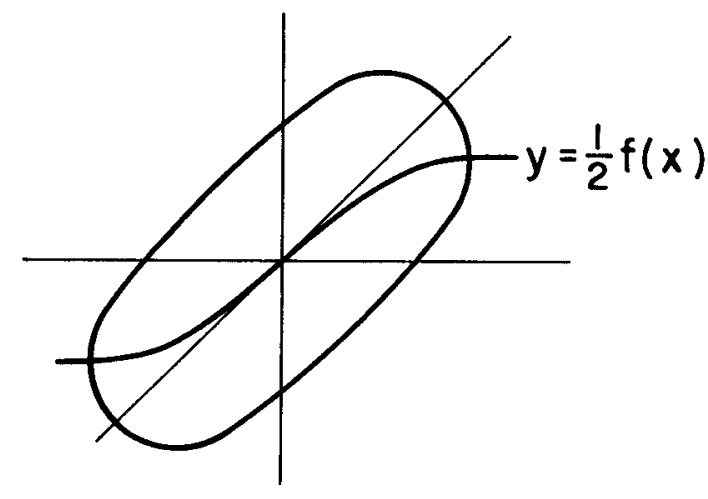

(b)

XBL679-5217

Fig. 2 
These curves the refore apply to the case of linear $f(x)$, a result already well known but interesting to derive in a new way. If $b<0$, the "invariant lines" are ellipses (or circles at $b=-1$ ) and the parameter a can have any positive value. If $b>0$ the lines are hyperbolas, and the parameter a is allowed both positive and negative values, giving both sets of branches of the hyperbolas. Thus a family of "invariant lines" resulting from a single $f(x)$ is generated by the variation of a single parameter. Whether such families can exist in more complicated cases remains an open question.

\section{A Generalization}

If $f(y)=\phi(y)+\psi(y)$ in (3), and $x=\phi(y)$, the transformation leads to $x^{\prime}=\psi^{-1}\left(y^{\prime}\right)$. This relation is reciprocal; a point on $x=\psi(y)$ goes to a point on $x^{\prime}=\phi^{-1}\left(y^{\prime}\right)$. The case considered in (3) corresponds to putting $\psi=\phi^{-1}$. Another case of interest occurs when $\phi^{-1}=\phi$ and $\psi^{-1}=\psi$. Geometrically, this means that $\phi$ and $\psi$ are curves with reflection symmetry about the positive diagonal, each crossing the diagonal once, as illustrated in Fig. 3a. The transformation moves a point from $\phi$ to $\psi$, the next iteration returns it to $\phi$, and so on. The two curves a re now second-order invariant lines and their intersections are second-order fixed points. These lines are again a solid barrier against the escape of points from the interior.

Closed curves can also be made by requiring that $\phi$ and $\psi$ join smoothly at their intersections, as in Fig. 3b. Now $\phi$ and $\psi$ can be considered together as forming a single curve, which forms a first-order "invariant line" of the type of Fig. $2 \mathrm{~b}$. In $3 \mathrm{~b}$, it has been given a shape such that $\frac{1}{2} f(x)$ at the center is sloping downward faster than the negative diagonal, giving a fixed point with oscillary instability at the origin. Here we have a particle confined forever in a region with no stable first-order fixed point! When the figure is also symmetrical about the negative diagonal, second-order fixed points occur at the intersection of $\frac{1}{2} f(x)$ with the negative diagonal. In this case, the outer ones are stable (the stable motion consists of jumping back and forth between these points) while the central one is unstable. From the origin there will proceed four secondorder invariant lines, which must not cross the smooth outer boundary. Whether these will succeed in joining smoothly, or whether they will fill the interior with a snaky tangle, remains an open question.

\section{Construction of Invariant Lines}

We have seen that a set of invariant lines has two symmetries, 'mirror symmetry about the positive diagonal and vertical symmetry above and below the curve $y=\frac{1}{2} f(x)$. (The transformation is equivalent to successive performance of these symmetry operations.) If the invariant lines 


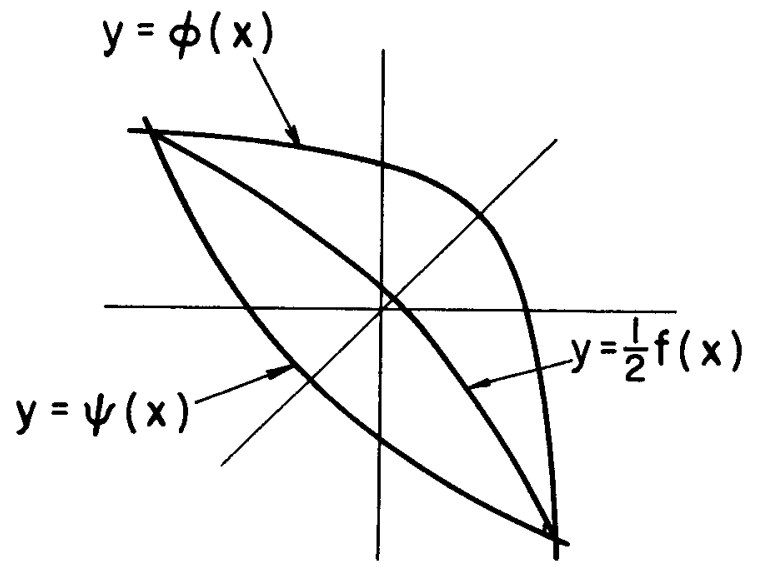

(a)

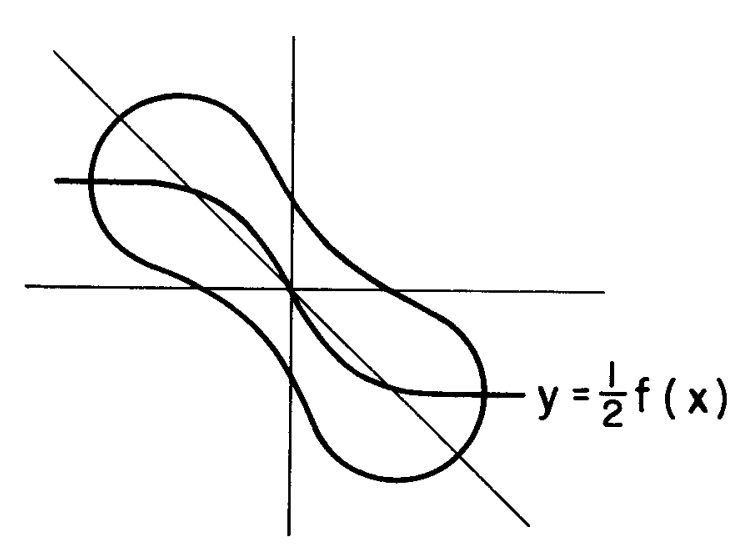

(b)

XBL679-5218

Fig. 3 
are given, $f(x)$ is easily constructed. A closed invariant boundary having the diagonal symmetry can be drawn arbitrarily so long as it is not more than double-valued; if it is there is a requirement of self-consistency in that the various branches must lead to the same $\frac{1}{2} f(x)$, and the curves cannot be drawn arbitrarily.

The inverse problem of finding the invariant lines from a given $\frac{1}{2} f(x)$ is not soluble in such a simple way. The only approach seems to be that used in computing invariant lines, to start at a fixed point and build from there. The construction described here is closely related to the computation procedure, but is useful in visualizing the process. It is illustrated in Fig. 4. One starts with a computed finite segment, marked 1. (The construction in principle could be started from a very small straight segment. $)$ Diagonal reflection gives segment 2. Vertical reflection of 2 repeats 1 and adds 3. From here on, alternate performance of the two kinds of reflections leads to segments $4,5,6, \cdots$ Segment 6 , in the case drawn, intersects $\frac{1}{2} f(x)$. The next two reflections lead to 7 and 8 , completing the first two loops. The symmetry making their areas equal is apparent. For further iterations complete loops can be used. Two additional loops are shown as dotted lines. Both types of reflection preserve areas, in consistency with the unity Jacobian of the transformation. If it had happened that segments 7 and 6 had joined smoothly, one would have had a case like that in Fig. 2a. This occurs if segment 6 has a vertical tangent where it intersects $\frac{1}{2} f(x)$. The property of joining smoothly clearly depends on an overall property of $\frac{1}{2} f(x)$, which could in principle be described by a single parameter, but it is not clear how this parameter can be determined except by "experiment" with a computer.

If there are two unstable fixed points bracketing a stable fixed point, the construction should be started at both points The invariant lines proceeding from the two points can join smoothly, or they can intersect, then systems of loops are started that move toward both fixed points.

\section{Discussion}

Some of the questions asked in the Introduction have been answered at least partially. Methods of finding lens functions that lead to wide classes of a rbitrarily chosen invariant boundaries have been found. The inverse problem remains at the mercy of the computer. However, it seems likely that the adjustment of a single parameter can convert a system without indefinite stability into one with indefinite stability, which fact may have practical importance. It is obvious that regions of indefinite stability can exist inside regions with only short-term stability, since one can find a function $\frac{1}{2} f(x)$ to give a prescribed smooth closed boundary, then extend it beyond, toward fixed points, in such a way that the invariant lines do not meet smoothly. No prescription is given for finding whether, inside a closed invariant boundary, there exist other closed invariant boundaries, or whether the motion inside is ergodic. 


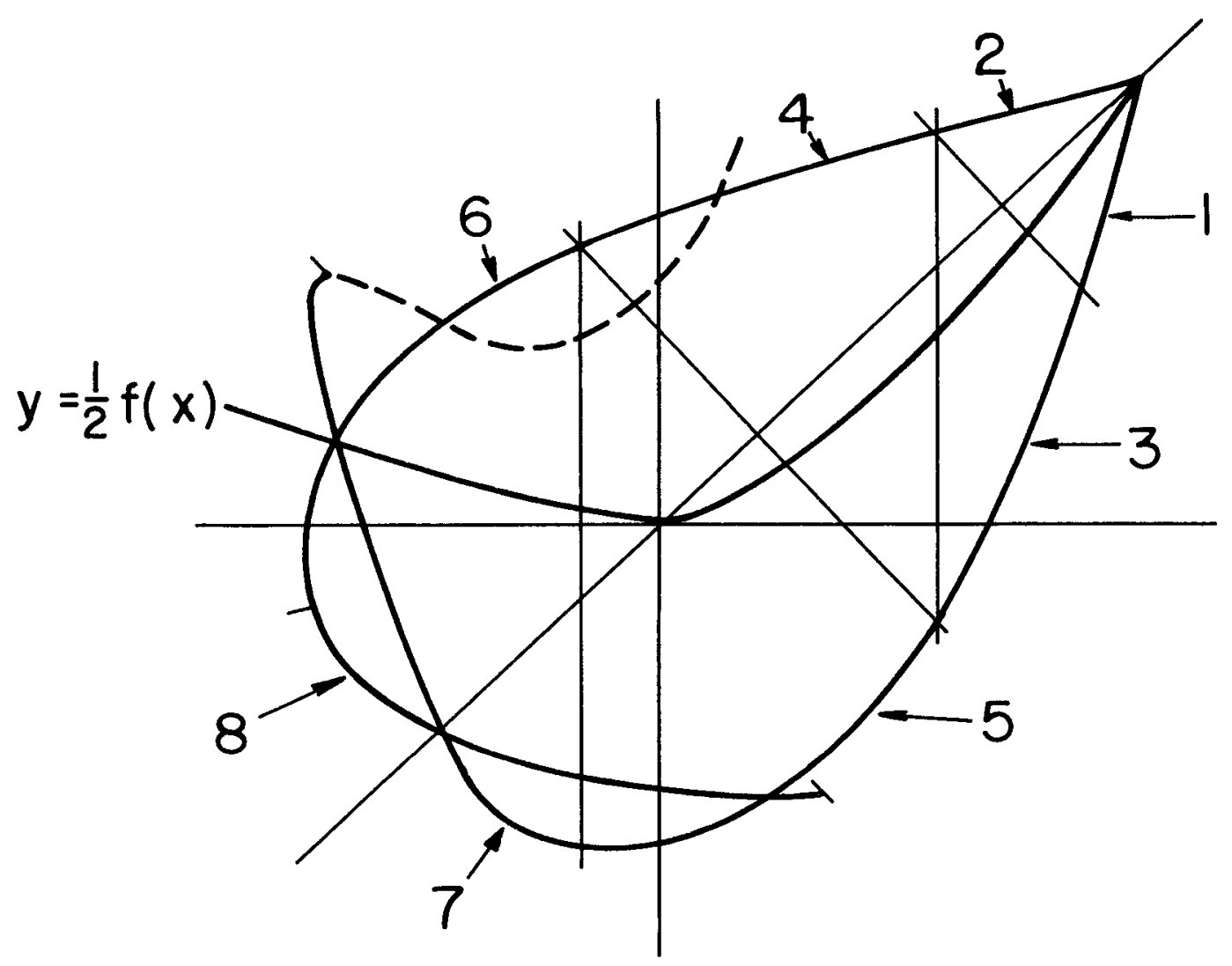

XBL679-5219

Fig. 4 
This report was prepared as an account of Government sponsored work. Neither the United States, nor the Commission, nor any person acting on behalf of the Commission:

A. Makes any warranty or representation, expressed or implied, with respect to the accuracy, completeness, or usefulness of the information contained in this report, or that the use of any information, apparatus, method, or process disclosed in this report may not infringe privately owned rights; or

B. Assumes any liabilities with respect to the use of, or for damages resulting from the use of any information, apparatus, method, or process disclosed in this report.

As used in the above, "person acting on behalf of the Commission" includes any employee or contractor of the Commission, or employee of such contractor, to the extent that such employee or contractor of the Commission, or employee of such contractor prepares, disseminates, or provides access to, any information pursuant to his employment or contract with the Commission, or his employment with such contractor. 